\title{
The "minimalist approach" for transfemoral transcatheter aortic valve replacement: Should we land at an airport without a fire department?
}

\author{
Torsten Doenst, MD, PhD, ${ }^{a}$ and P. Christian Schulze, $\mathrm{MD}, \mathrm{PhD}^{\mathrm{b}}$ \\ From the ${ }^{\mathrm{a}}$ Department of Cardiothoracic Surgery, University Heart Center Thueringen and the ${ }^{\mathrm{b}}$ Divisions of \\ Cardiology, Angiology, Pneumology, and Intensive Medical Care, Department of Internal Medicine 1, \\ Friedrich-Schiller-University of Jena, Jena, Germany. \\ Disclosures: Authors have nothing to disclose with regard to commercial support. \\ Received for publication Nov 27, 2015; accepted for publication Nov 30, 2015; available ahead of print Jan 14, \\ 2016 \\ Address for reprints: Torsten Doenst, MD, PhD, Department of Cardiothoracic Surgery, Friedrich-Schiller- \\ University of Jena, Erlanger Allee 101, 07747 Jena, Germany (E-mail: doenst@ med.uni-jena.de). \\ J Thorac Cardiovasc Surg 2016;151:1030-1 \\ $0022-5223 / \$ 36.00$ \\ Copyright (c) 2016 by The American Association for Thoracic Surgery \\ http://dx.doi.org/10.1016/j.jtcvs.2015.11.063
}

The advent of transcatheter treatment of aortic valve stenosis has expanded the therapeutic options and changed both the diagnostic and therapeutic approaches to patients with this disease. Spearheaded by interventional cardiologists, its introduction has also been accompanied by heated debates regarding the administrative structure and the personnel required to perform this new treatment with the highest degree of safety and procedural success. ${ }^{1,2}$ The establishment of so-called "heart teams" consisting primarily of cardiologists and cardiac surgeons that jointly see patients and decide on the treatment options as well as the joint conduct of the procedure in specifically equipped hybrid operating rooms is the current standard of care in most, if not all, major institutions. ${ }^{2}$

Recently, arguments have been raised that the transfemoral approach for transcatheter aortic valve replacement (TAVR) may be equally well performed in the cardiac catheterization laboratory, possibly even in the absence of cardiac surgical support and backup. ${ }^{1,3}$ Protocols have been developed to avoid intraprocedural transesophageal echocardiography, with local anesthesia and moderate sedation to avoid intubation of the patient. ${ }^{4} \mathrm{~A}$ " minimalist approach" has been adopted by several centers, where interventional-structural cardiologists perform procedures without proper surgical backup and vice versa, where surgeons perform transfemoral TAVR without proper interventional cardiology backup (unpublished observations). ${ }^{4}$ As the field is developing, with ever-increasing speed defining the future with broad implications, we need to ask critically which is the right and safest approach.

We compare the situation to that landing an airplane. Although it is possible to land airplanes on almost any stretch of land given the fact that a specific length of suitable ground is present, the requirements for landing commercial airplanes safely differ substantially. Which commercial airplane would be allowed to take off if the intended landing faa.gov/)?

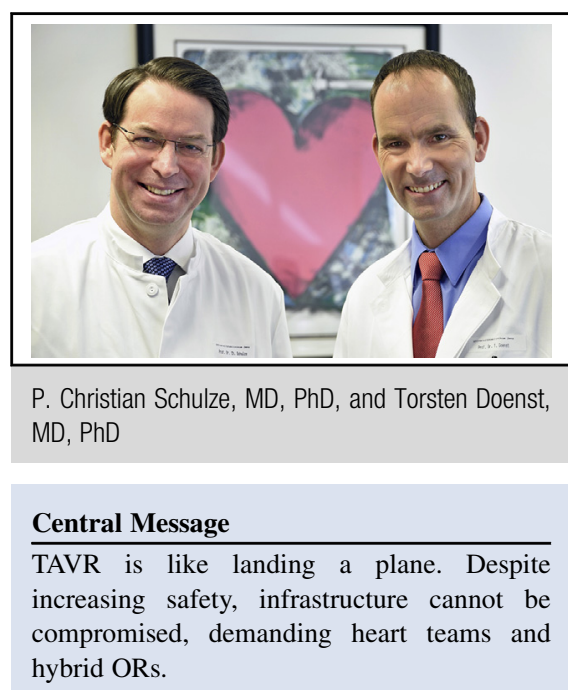

See Article page 1026.

airfield did not fulfill all the requirements of the national aviation agencies (the availability of the fire department would only be one of the requirements, see http://www.

Although the rate of intraprocedural complications contributing to morbidity and mortality is falling significantly with decreasing sheath sizes, better preprocedural imaging, well-defined intraoperative monitoring, and, most importantly, a distinct maturation of both devices and operator experience, significant morbidity is still associated with this procedure in both the high-risk and intermediate-risk populations. ${ }^{5}$

In this issue of the Journal, Greason and colleagues have addressed this issue, attempting to predict complications and to select those patients suitable for a minimalist approach. $^{6}$ They demonstrated in their series of 215 patients undergoing transfemoral TAVR that, despite a heart team discussion of patients and specific selection for transfemoral suitability, $10 \%$ of patients had significant intraoperative complications. More importantly, the type of complication was not predictable, and the complications included some that required immediate surgical intervention and would not have been easily addressed in the catheterization laboratory (such as major vascular injury in half of the complications, cardiac tamponade, or ventricular valve embolization). Although it is not 
specifically stated in the article, it appears that the procedures were performed in a hybrid operating room, and management of the complications described was mainly successful, because the perioperative mortality was very low at only $0.9 \%$. How a delay in dealing with the complications would have influenced outcomes is beyond the scope of the study, but it is a question that must be answered by those favoring the minimalist approach. Greason and colleagues ${ }^{6}$ conclude that patient and valve characteristics are not predictive of significant intraoperative morbidity but only suggest that this finding has implications regarding patient selection for the minimalist approach without further defining a position. The study is limited by a relatively long study period (2008-2015) that has been characterized by a dramatic development in this field as mentioned and likely changes in the rates of complications with time.

Despite the limited number of patients in this study, however, the results strongly argue that the process of identification of patients who can undergo transfemoral TAVR in the catheterization laboratory is complex and likely inconclusive, as some complications might occur in every patient. Because patient safety is our primary goal and the potential to intervene surgically immediately has to be guaranteed to everyone, there is no doubt that a hybrid operating room with a combined team of trained interventional and structural cardiologists and cardiac surgeons supported by cardiac anesthesiologists is the only answer.
If TAVR would truly be comparable to a commercial flight, this discussion would be unlikely to occur. There's a fine but decisive difference between pilots and cardiac surgeons and cardiovascular interventionalists-the members of the surgeon-cardiologist team usually survive the catastrophe.

\section{References}

1. Mylotte D, Head SJ, Kappetein AP, Piazza N. TAVI at institutions without cardiovascular surgery departments: why? EuroIntervention. 2014;10: $539-41$.

2. Nishimura RA, Otto CM, Bonow RO, Carabello BA, Erwin JP III, Guyton RA, et al. American College of Cardiology/American Heart Association Task Force on Practice Guidelines. 2014 AHA/ACC guideline for the management of patients with valvular heart disease: a report of the American College of Cardiology/ American Heart Association Task Force on Practice Guidelines. J Am Coll Cardiol. 2014;63:e57-185. Erratum in: J Am Coll Cardiol. 2014;63:2489.

3. Eggebrecht H, Mehta RH, Haude M, Sack S, Mudra H, Hein R, et al. Transcatheter aortic valve implantation (TAVI) by centres with and without an on-site cardiac surgery programme: preliminary experience from the German TAVI registry. EuroIntervention. 2014;10:602-8.

4. Jensen HA, Condado JF, Devireddy C, Binongo J, Leshnower BG, Babaliaros V, et al. Minimalist transcatheter aortic valve replacement: the new standard for surgeons and cardiologists using transfemoral access? J Thorac Cardiovasc Surg. 2015;150:833-9.

5. Walther T, Hamm CW, Schuler G, Berkowitsch A, Kötting J, Mangner N, et al; GARY Executive Board. Perioperative results and complications in 15,964 transcatheter aortic valve replacements: prospective data from the GARY registry. J Am Coll Cardiol. 2015;65:2173-80.

6. Greason KL, Pochettino A, Sandhu GS, King KS, Holmes DR. Transfemoral transcatheter aortic valve insertion-related intraoperative morbidity: Implications of the minimalist approach. J Thorac Cardiovasc Surg. 2016;151:1026-9. 